\title{
A New Framework for Request-driven Data Harvesting in Vehicular Sensor Networks
}

\author{
Louiza Mansour \\ LSI, Computing Department, USTHB University, Algiers, Algeria. \\ E-mail: louiza.mansour@yahoo.fr \\ Samira Moussaoui \\ LSI, Computing Department, USTHB University, Algiers, Algeria. \\ E-mail: moussaoui_samira@yahoo.fr
}

Received: July 14, 2013 Accepted: November 10, $2013 \quad$ Published: December 31, 2013

DOI: 10.5296/npa.v5i4.3155

URL: http://dx.doi.org/10.5296/ npa.v5i4.3155

\begin{abstract}
Vehicular Sensor Networks (VSNs) are an emerging network paradigm, suitable for various applications in smart-intelligent-vehicular network making use of vehicles' sensors as data sources and Inter-Vehicle Communication systems for the transmissions. The main issues in VSNs reside in the data harvesting and its dissemination in such a large-scale network, characterized by the frequent topology changes and network partitioning. To achieve this, an efficient collaboration between sensors and vehicles is required. This paper proposes a new multi-hop Collaborative Data Replicas Harvesting Protocol (CDRH) based on data replication on VSNs in an urban scenario. The aim of our proposal scheme is to achieve a high accessibility to a Request Data while maintaining a low level of channel utilization. The simulation results show that this protocol can achieve significant performance benefits.
\end{abstract}

Keywords: Vehicular Network, Vehicular Sensors Network, Smart road, Collection, Dissemination, Replication. 


\section{Introduction}

Over the past few years, advances in wireless networking and embedded computing have led to the "first generation" of wireless sensor networks, including some impressive field deployments [1, 2, 3, 4]. Usually, these are for monitoring or tracking applications characterized by low data rates and static deployments. Although a new emerging network paradigm appears, Vehicular Ad hoc NETworks (VANET) [5, 6]. Vehicular networks are a class of mobile networks in which vehicles are equipped with radio interfaces and are therefore able to communicate with an infrastructure (if existing) or other vehicles, to achieve safety, driving assistance, and entertainment.

Some applications [7] need to handle data obtained by sensors which are mounted on vehicles' on-board computers and on roadside infrastructures. In this case resulting networks are called Vehicular Sensor Networks (VSNs). A VSN can be built on top of a VANET by equipping vehicles with sensing devices. VSNs are emerging as a new tool for effectively monitoring the physical world, especially in urban areas where a high concentration of vehicles equipped with onboard sensors is expected [8].

Compared to traditional static sensors, a sensor node is not subject to processing, storage, and energy limitations. Moreover, a mobile sensor node improves sensing coverage with low costs. The two primary distinct features of vehicle networks are that i) vehicles can be highly mobile so an intermittent connectivity, and ii) their mobility patterns are more predictable than those of nodes in Mobile Ad hoc NETworks (MANETs) [9] due to the constraints imposed by roads, speed limits, and commuting habits. These specific characteristics generate a number of new research challenges (e.g. data dissemination, data aggregation, data collection, security and authentication ...) that need to be addressed for VSNs to be widely deployed. In this work, we focus on one of the most important topics in VSNs: data collection.

The typical scale of a VSN over a wide geographic area, the volume of generated data, important mobility of vehicles and the limited bandwidth make it infeasible to adopt traditional sensor network solutions $[10,11]$. The challenge is processing queries in this highly mobile environment and avoids broadcast storm problem [12] to overcome delay, overhead and accuracy. Some approaches have been proposed to deal data collection in VSNs.

In [5], an opportunistic dissemination is used to harvest meta-data. A cluster based approach is used in [8], and in [13] a data replication technique is used. These approaches have some constraints, as they limit their data harvesting schemes on limited area [5], use centralized access point [8] and a high-cost band for communication [6].

We propose a new scheme for disseminating and harvesting data in a smart VSN [14, 15] using multi-hop communication and data replication. To reduce overhead, our protocol takes advantage of the vehicle's motion by using a communication between vehicles. The aim of our proposition is to satisfy the requesting vehicle (collect a Request Data), and also make this data available for other future requesting vehicles. 
The rest of this paper is organized as follows. Section II presents the related work focused on data dissemination and data collection on VSNs. Section III describes our proposed protocol. The performance evaluation of this protocol is presented in Section IV. Finally, we conclude the paper and give some perspectives to our work in Section V.

\section{Related Work}

The majority data harvesting protocols realized for vehicular sensor networks are through a dissemination mechanism. Several dissemination protocols were proposed for VANETs. Concerning the type of the targeted applications, protocols could be sorted into two classes: (i) protocols for infotainment services [5] that have constraints related to the bandwidth, and (ii) protocols for emergency services [16] that have end-to-end delay and delivery ratio constraints.

Regarding the architecture communication used in the network, we can classify data dissemination into two classes: (i) Data Dissemination for the V2V (Vehicle to Vehicle) architecture, and (ii) Data Dissemination for the V2I (Vehicle to Infrastructure) architecture. The solutions based on a V2V communication use the communication techniques known in the ad hoc networks. In this approach vehicles form ad hoc networks by using wireless interfaces. The first issue of this approach is the Broadcast Storm problem [12] which can decrease significantly the rate of delivery of messages. The second issue that must these solutions take into account is the network partitioning. Among works using this architecture, we find: OAPB (Optimized Adaptive Probabilistic Broadcast) [17] and ODAM (Optimised Dissemination of Alarm Messages) [18]. In order to overcome the problems known in the $\mathrm{V} 2 \mathrm{~V}$ communication, solutions which use a propagation assisted by infrastructure points were proposed. But, they cause problems as scalability and cost. STEID (Spatio_Temporal Emergency Information Dissemination) [19] is an example of these protocols.

Different approaches for data dissemination in a VSN can be made: (i) Opportunistic dissemination, (ii) Geographical dissemination, (iii) Peer-to-peer dissemination, and (iv) Cluster-based dissemination. Some works, such as [8, 20], recommend the use of opportunistic dissemination of data, in which messages are stored in each intermediate node and forwarded to every encountered node, till the destination is reached. Thus the delivery ratio is improved. However, this kind of mechanisms is not suitable for non-delay tolerant applications (emergency services).

Due to the fact that end to end paths are not constantly present in a VSN, a geographic dissemination is used in $[13,21]$ by sending the message to the closest node toward the destination till it reaches it. Another way to do geographic dissemination is given in [8] where the authors show how to use geo-casting to deliver messages to several nodes in a geographical area. In a P2P solution, the source node stores the data in its storage device and do not send them in the network till another node asks for them. In [13], such architecture is proposed for delay tolerant applications.

For a better delivery ratio and to reduce broadcast storms, a message has to be relayed by 
a minimum of intermediate nodes to the destination. To do so, nodes are organized on a set of clusters, in which one node or more (Cluster Head) gathers data in his cluster and send them after to the next cluster. Cluster-based solutions provide less propagation delay and high delivery ratio with also bandwidth fairness. In [16] the authors use a distributed clustering algorithm to create a virtual backbone that allows only some nodes to broadcast messages and thus, to reduce significantly broadcast storms.

To collect sensed data, a number of models have been widely used in wireless sensor networks [22, 23]. However, these approaches are less efficient in VSNs because of i) the typical scale of a VSN, ii) the volume of generated data, and iii) the vehicles mobility. Data harvesting is considered as the first step of most of VSN applications. The aim of data harvesting in VSNs is to facilitate the two following steps in the VSN traditional life-cycle: i.e. data search and data dissemination.

We can classify Data collection on VSNs into two main categories: (i) Continuous Data Collection Protocols and (ii) Request-Driven Data Collection Protocols. In a Continuous Data Collection Protocol [6, 9, 10], vehicles are used us a source of information: (i) The vehicles send information issued from their on-board sensors to a server (sink) (ii) The server collects the data and treats them (iii) The server disseminates useful information to the interested vehicles. These kinds of protocols are used in monitoring applications like traffic monitoring, Parking management, Pollution monitoring ... In a Request-Driven Data Collection Protocol $[5,13]$; the data collection process is initialized by sending a Data Collection Request. The Data Collection Request can be sent either by a moving vehicle or a road infrastructure. This kind of protocols is used to collect a specific data in a defined time and area.

CarTel [8] is a mobile sensor computing system designed to collect, process, deliver, and visualize data from sensors located on vehicles. Each CarTel node uses a geographical dissemination to gather data; process sensor readings and deliver them to a central station (portal). CGP (Clustered Gathering Protocol) [6] is a cross-layered gathering, dissemination and aggregation protocol, based on a geographical clustering in a hybrid vehicular architecture (V2V: Vehicle to Vehicle and V2I: Vehicle to Infrastructure). It collects information from nodes, aggregates them and sends them to a provider via high cost links. The concept of ADCD [24] is based on the characterization of sensed information (i.e. based on its importance, location and time of collection) and the diffusion of this information accordingly. Furthermore, ADCD uses an adaptive broadcasting strategy to avoid overwhelming users with messages in which they have no interest. ADCD aims at reducing the generated overhead avoiding network congestions as well as long latency to deliver the harvested information.

In [5], authors propose a solution to disseminate and harvest data. Private vehicles (regular nodes) opportunistically and autonomously spread summaries of sensed data. A harvesting protocol is used by police agents (authority nodes) to build a distributed index of the mobile storage of sensed data. This index allows law enforcement agents to querying a huge database without centralization. In [13] a multi-hop geographic data dissemination method using data replication technique to collect sensor data in VSNs adopting a V2I 
communication is presented. In this solution, to achieve the required data to the requested vehicle, the only way to communicate is between static nodes, so we can consider the environment used like a traditional WSN.

In Road Probing [12], the Road Side Unit (RSU) initiates the probing process and selects the passing by vehicles as probes to collect traffic and environment information. The selected vehicles sense the requested data and forward it back to the RSU in a multi-hop fashion. In DB-VDG (Delay-Bounded Vehicular Data Gathering) [25], authors focus on data gathering based on VSNs in a urban scenario. The main novelty in this work is the exploitation of the time interval during which the data gathering process is active. DB-VDG uses the delay-bounded paradigm opting for either forwarding the data immediately or carrying them in memory while the vehicle moves, such as delay-bounded routing protocols [26]. This behavior aims at reducing forwarded messages and at aggregating data from different sources in a single node so as to include multiple data in a single message.

The aim of our proposal is to develop an efficient strategy to collect a required data. By efficient we mean that the process has to be able to collect data, with a low bandwidth communication cost. Our data collection protocol CDRH (Collaborative Data Replicas Harvesting protocol for VSNs) optimizes the bandwidth usage by minimizing the amount of vehicles that have to rebroadcast packets by using the same principle as DDT (Distance Defer Transfer Protocol) [27]. In DDT, only one vehicle is selected with each transmission to rebroadcast the message. In our approach, we try to satisfy a requesting vehicle and offer a high accessibility to the Request Data by using data replication. We take advantage of the vehicles' mobility, thus enable a collaborative harvesting by mobile and static sensor nodes.

\section{CDRH: Collaborative Data Replicas Harvesting protocol for VSNs}

Currently, some critical issues in VSNs, such as data transmission delay, packets overhead, and network connectivity are not completely solved. The presented protocols have some restrictive assumptions, as they suppose a data collecting schemes to only single-hop or short distance transmissions [5], or use relay stations and cellular communications to support multi-hop collection [6,8], which can be very costly and complex. However many of reported works are solutions to specific applications. The presented protocols use either a V2V communication [5] which can induce the broadcast storm problem, or a V2I communication $[8,13]$ which is expensive.

Until now, only a few research works have addressed the problem of data gathering in vehicular sensor networks. They have lead to solutions relying on push models, where potentially useful data are pushed towards vehicles $[8,12]$. All the dissemination protocols, and by extension every data collection relying on them, are generally not upon request. As a result, information is frequently broadcasted even if it is not necessary, leading to bandwidth waste. Moreover, since vehicles do not know which data will be relevant, they will tend to broadcast more than expected. To solve this problem, data collection has to be initiated from a request launched by a node (vehicle, base station, internet provider ...). 
In this work, we tackled the use of pull models in VSNs. Such models would allow users to send queries into the network in order to find a requested data. In order to propose such a query processing scheme, the main challenge addressed is to find the requested data and route it towards it recipient in a highly dynamic network. To solve this issue, we propose CDRH.

CDRH Collaborative Data Replicas Harvesting Protocol is a new scheme for disseminating and harvesting data in a smart VSN using multi-hop communication and data replication. The aim of CDRH is to satisfy vehicle's request and also allow accessibility to the data to other potential requesting vehicular nodes.

Our protocol has the capabilities to (i) avoid the waste of bandwidth by minimizing the amount of vehicles that have to rebroadcast received packets, take advantage of the vehicular nodes motion, use a request driven data collection and a delay-bounded data dissemination; (ii) Use a hybrid architecture to communicate, so our solution deals with the broadcast storms problem, cost and scalability issues; (iii) Use a replication process to make the data easy accessible.

\subsection{Protocol Environment}

We consider a vehicular sensor network using V2V and V2I communication in a urban or a highway area. The ITS (Intelligent Transports Systems) [28] applications aimed by this proposed protocol are general information services that's provides conviviality like recognize the nearby road situation, traffic conditions, environmental noise or air pollution level. On this environment, we assume that the IEEE 802.11p standard is used and the nodes are equipped with a GPS device for localization and synchronization. Each node (sensor nodes and vehicular nodes) creates and maintains a neighborhood list via beacon messages, and a replicas table. The collected data are replicated and stored locally in the replicas table.

\subsection{Protocol overview}

In this section, we detailed the proposed protocol from the request transmission to the data reception. The scheme will be divided into four parts: (i) Data Requesting Phase, (ii) Data Delivery Phase (iii) Data Replication Phase, and (iv) Data Sharing Phase.

When a vehicle node needs to collect a data, it sends a request packet. This packet contains vehicle's information (identifier, velocity $v(\mathrm{~m} / \mathrm{s})$ and coordinates). Then, three different cases can be observed:

- The required data is available on a neighborhood vehicular node: Upon receiving a query, the vehicular node tries to perform it locally in its database. In case of success, the data packet will be automatically sent to the source node. In this phase, the query spreads only because of the vehicle mobility. This communication decreases the packet overhead since there is a probability that another vehicle has before made the same request. After a timeout, if there is no response, the request is sent to a sensor node.

- The Request Data is available on a neighborhood static sensor node: In this case this sensor node sends straightforwardly the Data Packet to the vehicular node.

- The Request Data is available on a non-neighborhood static sensor node: In this case, the Request Packet is sent to the farthest static node. This static node (RS: Requesting 
Sensor) adds its identifier and its coordinates to the packet, and forwards it. The RS initiates a timer "Replication Timer" RepT (seconds) which will be used later for supervising the Data Replication process. The value of RepT will be the estimated time to retrieve the Data and to deliver it to the RS node. The Request Packet will be forwarded, until it reaches the node holding the Request Data.

At the reception of Request Packet, the node holding the Request Data sends the Data to the previous node on the Request Packet path, until it reaches the RS node. When the RS node receive the Data Packet, it saves it in his local replicas table and begun the replication process. The replication process depends on the requesting vehicle velocity $v$, the size of the Data $p$ and the sensors transmission rate $r$.

When the RS receives the Data, it broadcasts the packet to its neighbors, creating replicas of the Data. For restricting the flooding overhead, we use a Time-To-Live value TTL. Equation (1) gives the number of hops that the RV has moved while receiving the Data.

$$
v \times \operatorname{Rep} T \div d
$$

Where, $d$ is the distance separating two sensor nodes.

While equation (2) gives the distance (by hops) the RV has traveled while the Data is transmitted to the vehicle.

$$
v \times(p \div r) \div d
$$

From the two equations (1) and (2), we can determine the appropriate TTL value.

$$
T T L=[v \times R e p T \div d+v \times(p \div r) \div d]
$$

Depending on the TTL value, RS broadcasts the Data Packet to its neighbors and sends a unicast message to the farthest static node on the requester vehicular node's direction. This node will do as the RS until expiration of the TTL. When the Data reaches a sensor node close to the initiator vehicular node RV, it sends it to the RV.

In an urban environment there is a high probability that a number of vehicular nodes request for the same data, so we use the replicas created in the previous step. After the replicas are made by a request from the first vehicular node; other following vehicular nodes can benefit from the data availability and access it directly.

\subsection{CDRH and intersections}

In an urban environment, and during the request propagation, the request packet may probably cross an intersection. To deal with this case, a simple way is that when the packet gets to an intersection node, it will be broadcasted (flooding). Simple flooding causes the broadcast storm problem which produces an excessive bandwidth use and, it increases the end to end delay and packet loss ratio. In our solution, another approach is used. In an intersection point, CDRH defines a set of nodes called Intersection Nodes. Each Intersection Node harvests data in its road-segment in a permanent way. When a vehicular node request 
for the data, it can found it more easily, because it's probably already collected by at least one of the intersection node.

In CDRH, a data aggregation mechanism is used because the exchanged data volume is important in a permanent data harvesting mode. When a node receives a Data packet, it compares the ID-Data field of the received packet with those of his Data packets. If any Data packet saved in its Replicas table has the same ID-Data value, this packet is dropped; else the data packet is saved in the Replicas table. The same process is used by the other node, till the intersection node is reached. By using this aggregation technique, the intersection node will receive one copy of each collected Data.

\section{Comparison and Performances Evaluation}

The simulations consider a vehicular network with a number of vehicles between 80 and 120 and 121 sensor nodes distributed uniformly on each side of the road in a $2100 * 2100 \mathrm{~m} 2$ area. The distance between each node is set to $200 \mathrm{~m}$. The generation frequency of beacon messages is set to 10 messages /s. The average speed of vehicles $\mathrm{v}$ is between $0 \mathrm{~m} / \mathrm{s}$ and 30 $\mathrm{m} / \mathrm{s}$ and the size of the data $\mathrm{p}$ is set to 1000 bytes and these of the request and beacons, is set to 200 bytes. The data is supposed existed in one sensor node. The distance separating the vehicular node initiator of the request and the sensor node holding the data is set between $45 \mathrm{~m}$ and $1800 \mathrm{~m}$. We evaluate CDRH performance through extensive simulations using NS-2 [29]. For realistic mobility generation, we use SUMO (Simulation of Urban Mobility) [30] and MOVE (Motor Vehicle Emission Simulator) [31]. The simulation results are an average of more than twenty executions.

To evaluate CDRH performances, we mainly focused on (i) the necessary number of hops for data collection, (ii) the Response Time and (iii) the Packets Overhead.

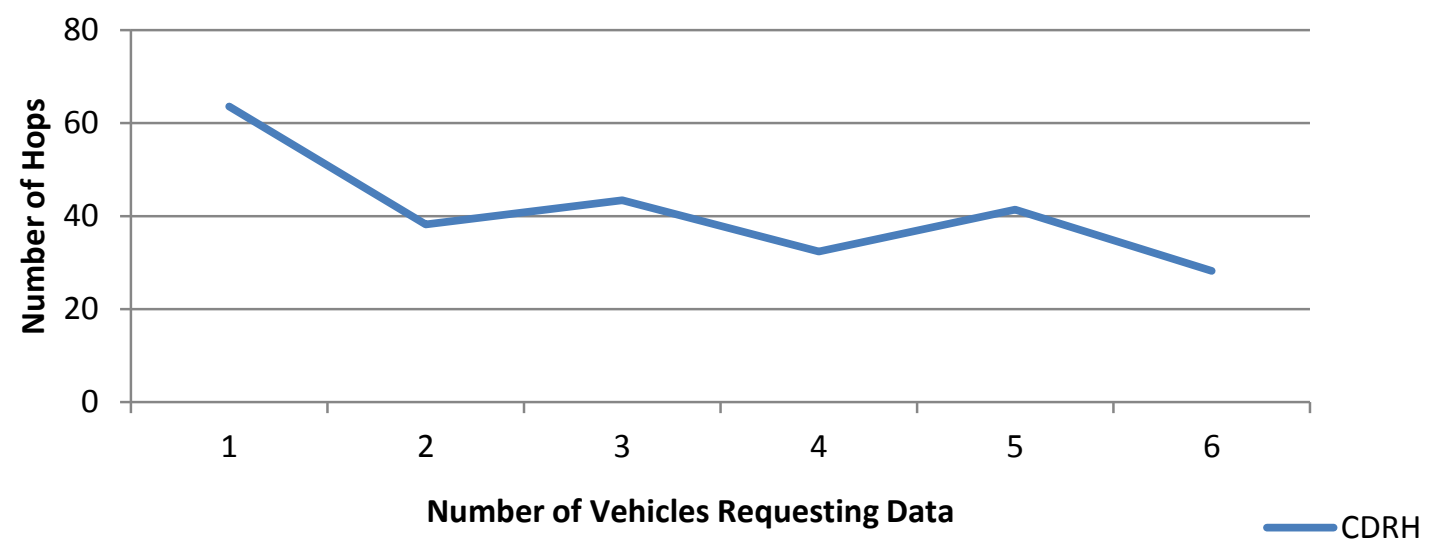

Figure 1. Number of Hops / Number of Vehicular Nodes Requesting for Data.

Figure 1 shows the number of hops it takes to deliver the Request Data depending on the number of requesting vehicles. The $\mathrm{x}$-axis was configured to the number of vehicles 
requesting for the data, which varies from 1 to 6 . It can be seen that in the proposed scheme more the number of vehicular nodes requesting the data is important, more the number of hops required to deliver the data decrease. This is because more copies of the Request Data are made as the requesting vehicular nodes increase, so the vehicular nodes can access to the data more easily even if it's there are other previous requester vehicles to the data. In effect, the Request Data can be on a neighbor vehicle or sensor. This is the advantage of data replication, where the vehicular node can harvest the data on a node containing the replicas of this data.

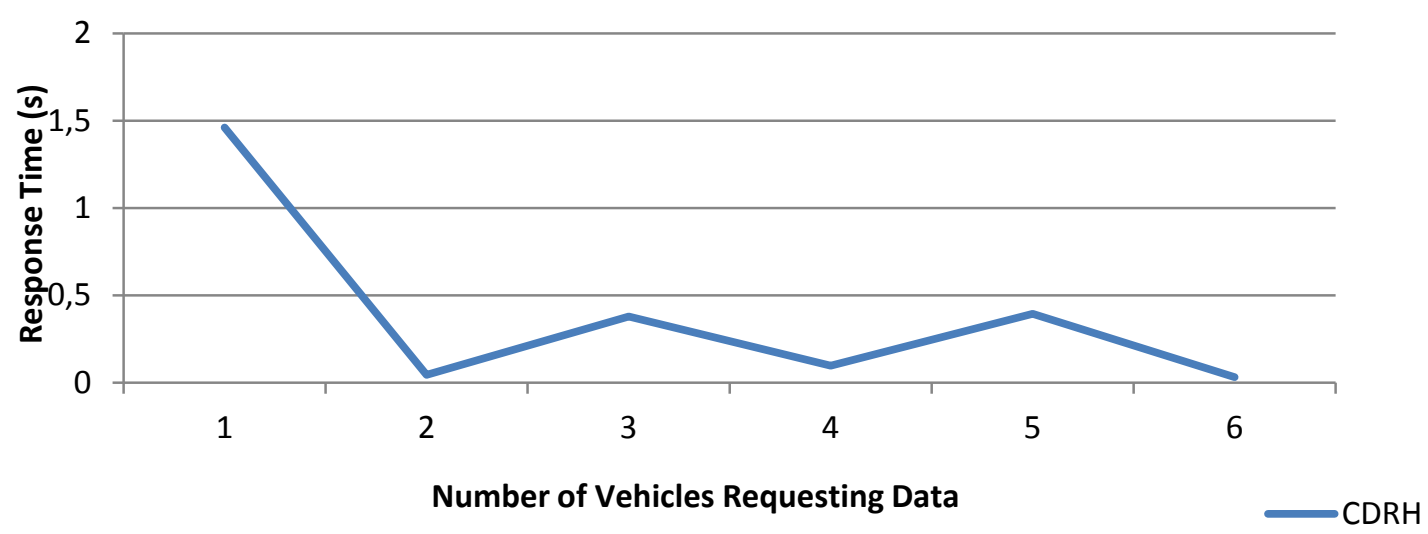

Figure 2. Time of Response / Number of Vehicular Nodes Requesting for Data.

Figure 2 shows the response time to deliver the data from sending the first request packet to receiving the last data packet depending on the number of vehicles requesting the data. The $\mathrm{x}$-axis was configured to the number of vehicles requesting for the data, which varies from 1 to 6 . It can be seen that more the number of vehicle requesting the data is important, more the delivery time decrease. This is due that vehicular nodes can reach the data more quickly, because more copies are made when the number of vehicular nodes requesting the data is important. So the vehicular nodes can get the data from a node containing a copy of the data and the requests have not to reach the source node. Also, the communication between vehicular nodes helps to get the data directly without any replication process used.

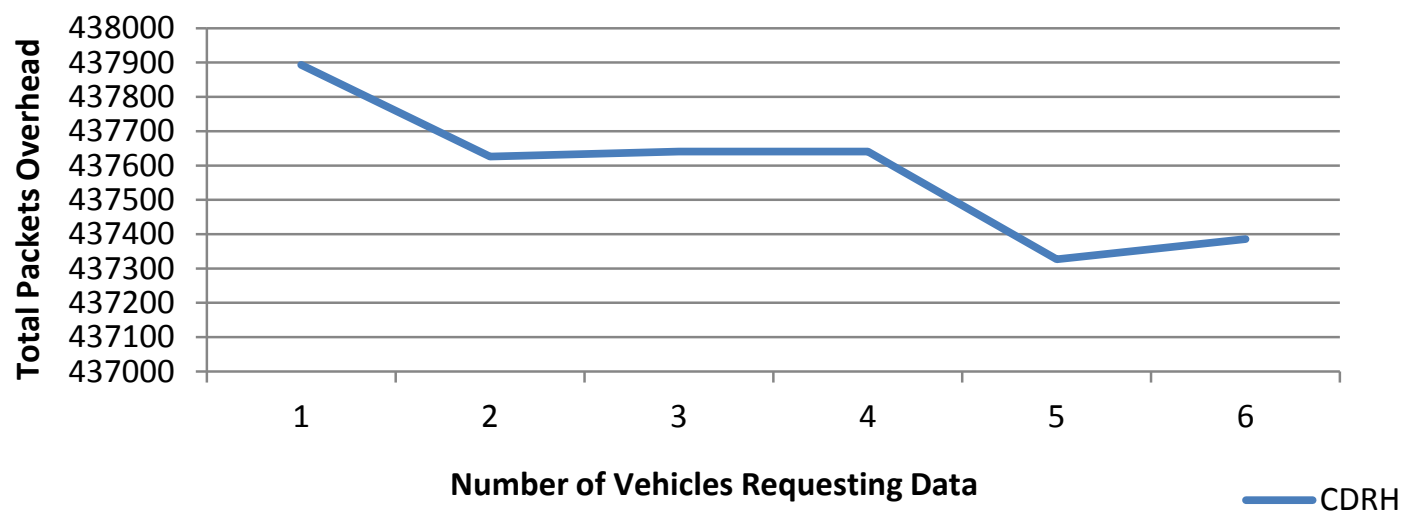

Figure 3. Total Packets Overhead / Number of Vehicular Nodes Requesting for Data. 
Figure 3 shows the total packets overhead generated by our proposition. The x-axis was configured to the number of vehicles requesting for the data, which varies from 1 to 6 . We can observe that more the number of vehicles requesting for the data increase, more the packets overhead is less important due that the vehicular node found the data more quickly so there is no need to exchange more packets. Also, the data sharing technique used by our proposition reduces further packets overhead, as no replicas are created.

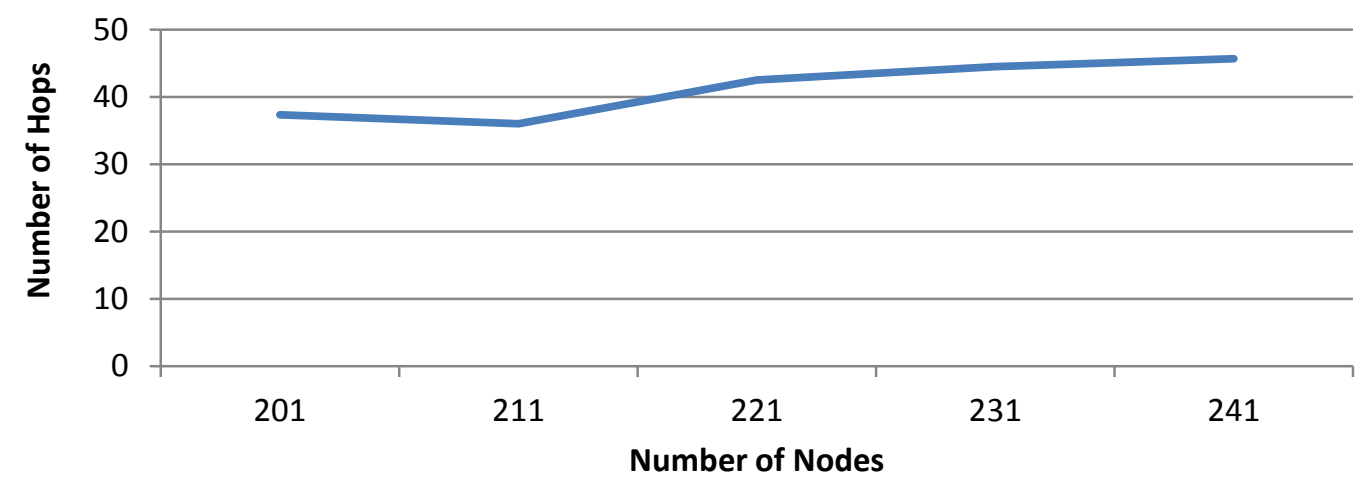

Figure 4. Number of Hops / Number of Nodes.

The $\mathrm{x}$-axis was configured to the number of nodes in the network, which varies from 201 to 241. Increase the number of nodes causes more packets exchange in the network, causing collisions. Therefore, in figure 4, we can observe an increase of number of hops in the proposed scheme as the number of nodes increases. But, when the number of vehicle became important, we can observe that the response time decreases again because replicas of the data are created during previous requests.

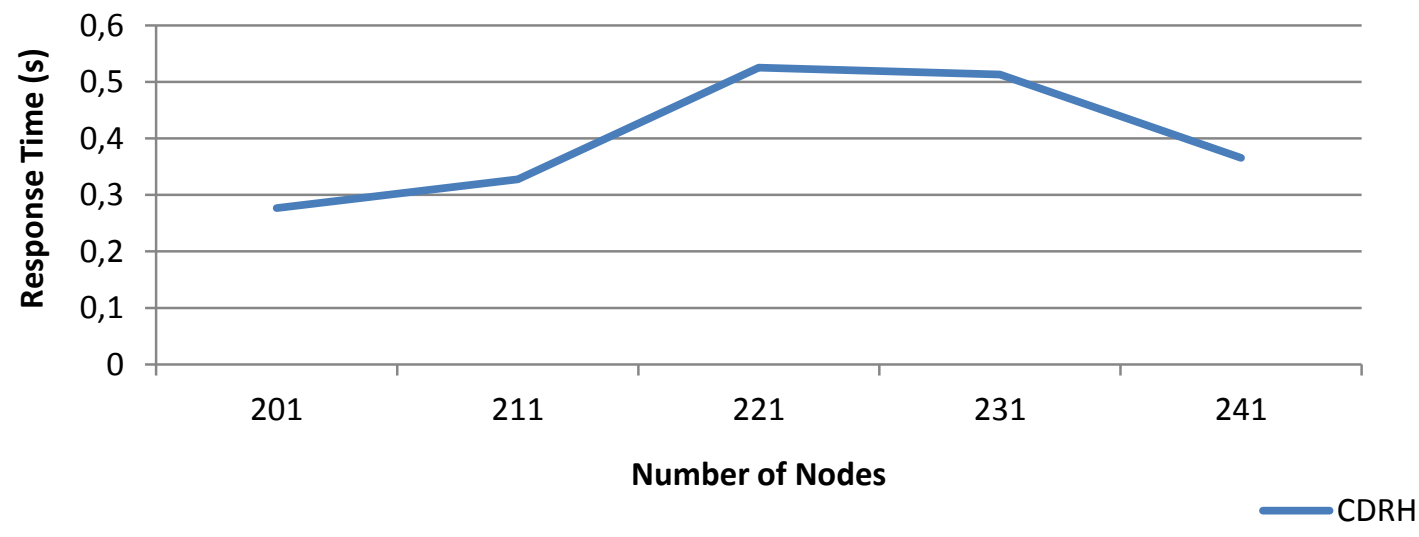

Figure 5. Time of Response / Number of Nodes.

We can see in figure 5 that more the number of vehicular nodes increase, more the response time decrease. That is due mainly that more communication between vehicular nodes is used. Caused by the beaconing messages, the total packets overhead generated by our proposition increase gradually as the number of nodes increase (figure 6). 


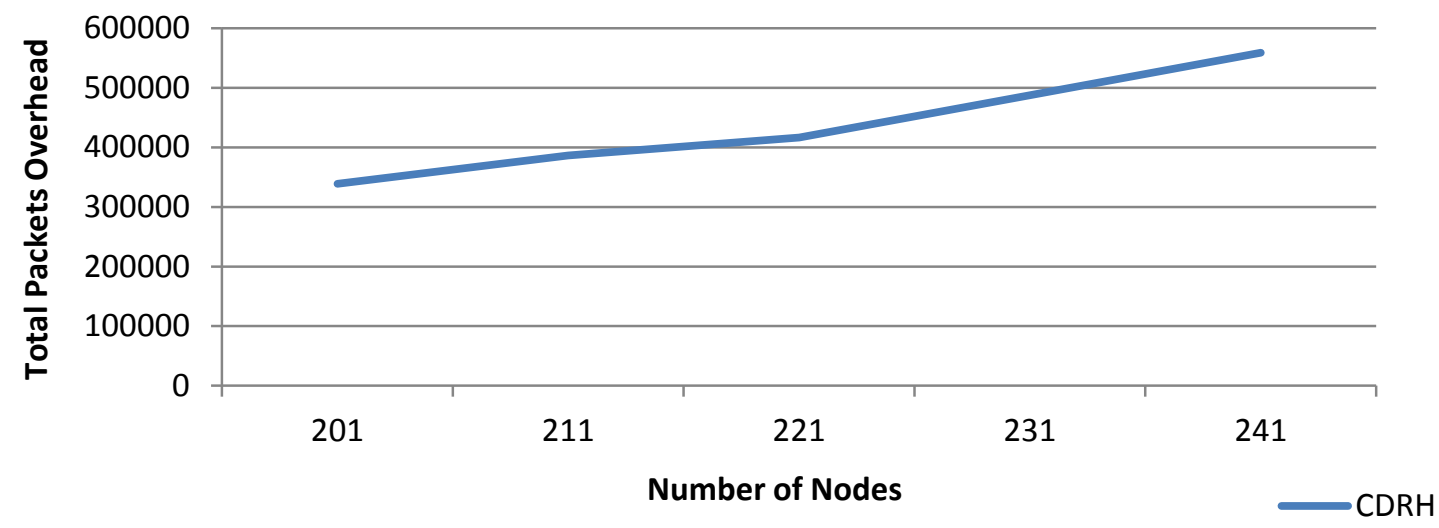

Figure 6. Total Packets Overhead / Number of Nodes.

Figures 7 and 8 show a comparison study made to demonstrate the benefits gained in terms of number of hops (figure 7) and response time (figure 8).

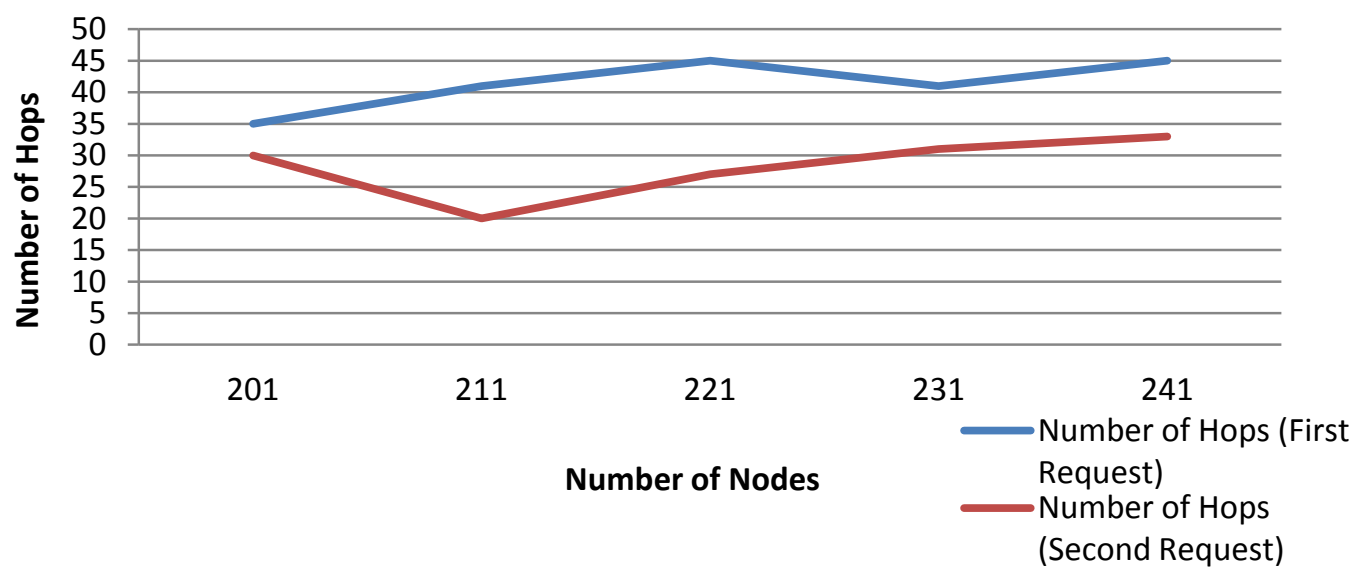

Figure 7. Number of Hops / Number of Nodes.

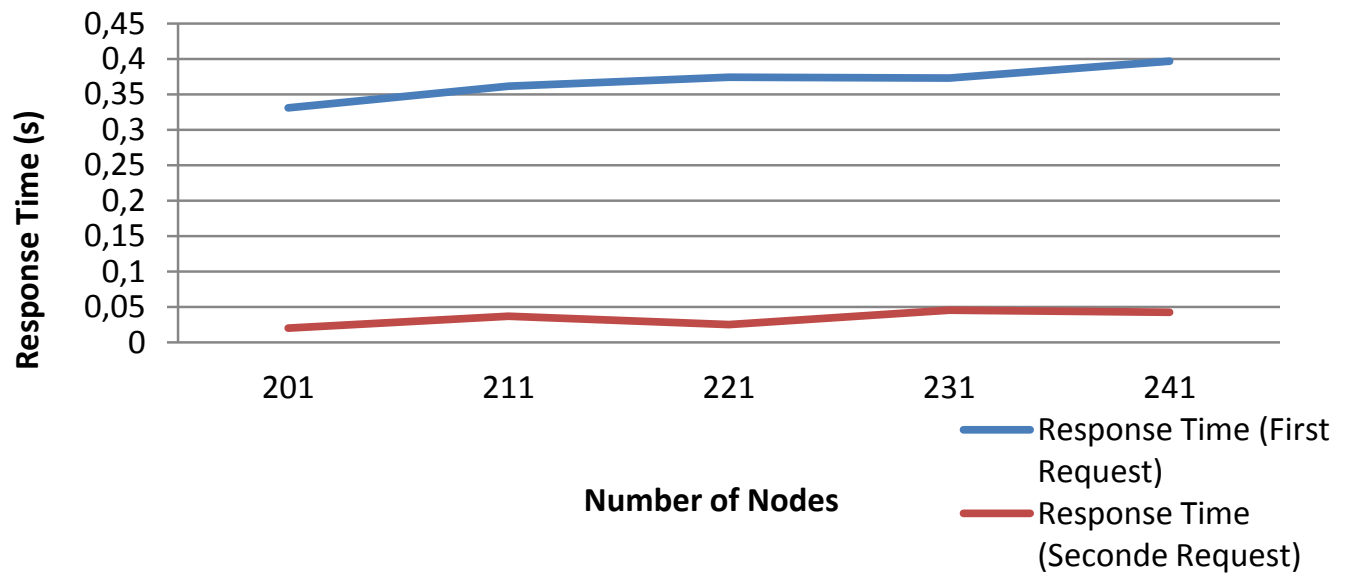

Figure 8. Response Time / Number of Nodes. 
It can be seen that the number of hops needed to collect the data for the second request is lower than this of the first one. That can be explained that the data is founded quickly and more easily due to the replicas created from the first request. The same results are observed for the response time (figure 8).

In table 1, we compare our protocol to other data collection protocols.

Table 1. Comparative study of CDRH with other Data Collection Protocols.

\begin{tabular}{|c|c|c|c|c|c|}
\hline Frotocol & $\begin{array}{c}\text { Network } \\
\text { Architecture }\end{array}$ & $\begin{array}{c}\text { Data } \\
\text { Collection }\end{array}$ & $\begin{array}{c}\text { Data } \\
\text { Aggregation }\end{array}$ & $\begin{array}{c}\text { Road } \\
\text { Environment }\end{array}$ & $\begin{array}{c}\text { Delay-bounded } \\
\text { Data } \\
\text { Collection }\end{array}$ \\
\hline CDRH & V2V+V2I & $\begin{array}{c}\text { Pull-based and } \\
\text { Push-based in } \\
\text { intersection } \\
\text { points }\end{array}$ & Yes & $\begin{array}{c}\text { Urban + } \\
\text { Highway }\end{array}$ & Yes \\
\hline MDHR & V2I & Pull-based & Yes & Urban & No \\
\hline CGP & V2V+V2I & Push-based & Yes & Urban + & Highway \\
\hline DB-VDG & V2V+V2I & Pull-based & Yes & Urban & Yes \\
\hline ADCD & V2V & Push-based & No & Urban & No \\
\hline
\end{tabular}

Among the protocols studied in the related work section, the MDHR protocol is selected for the comparison study because it is the only pull-based data collection protocol which uses the data replication process.

In figures 9, 10 and 11, we compare CDRH performance's with MDHR. The x-axis was configured to the number of requesting vehicles in the network, which varies from 1 to 6 .

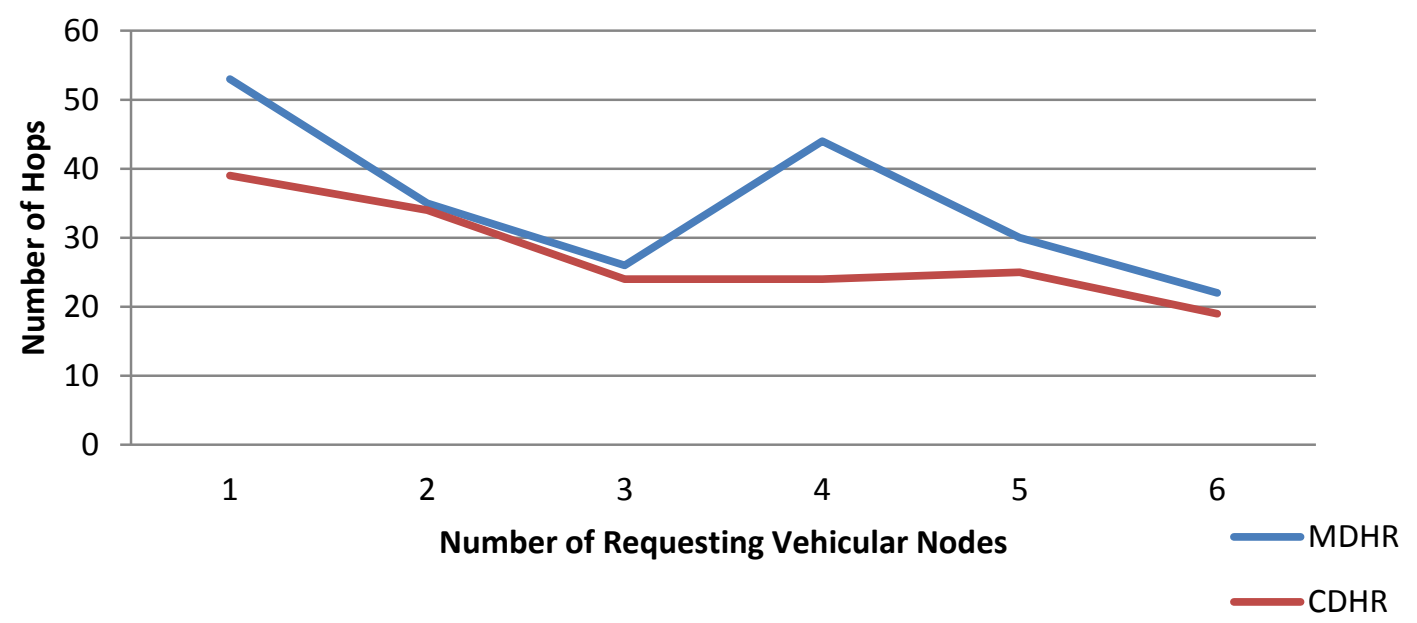

Figure 9. Number of Hops / Number of Vehicular Nodes Requesting for Data.

Figure 9 show the comparison of the average number of hops it takes from sending the first request packet to receive the last data packet. It can be seen that the proposed scheme has 
advantages over the MDHR protocol. This is can be explained that the data collection strategy used in CDRH (the use of DDT mechanism) can collect the data where ever the data is (the dissemination mode used can reach farthest nodes and cover a larger area), and the opportunistic communication used between vehicular nodes can satisfy a request in just one hop. Whereas, in MDHR, even if the data is on a neighborhood vehicular node, it's cannot be delivered in a single hop, and a new costly process of research and delivery of the data is initiated.

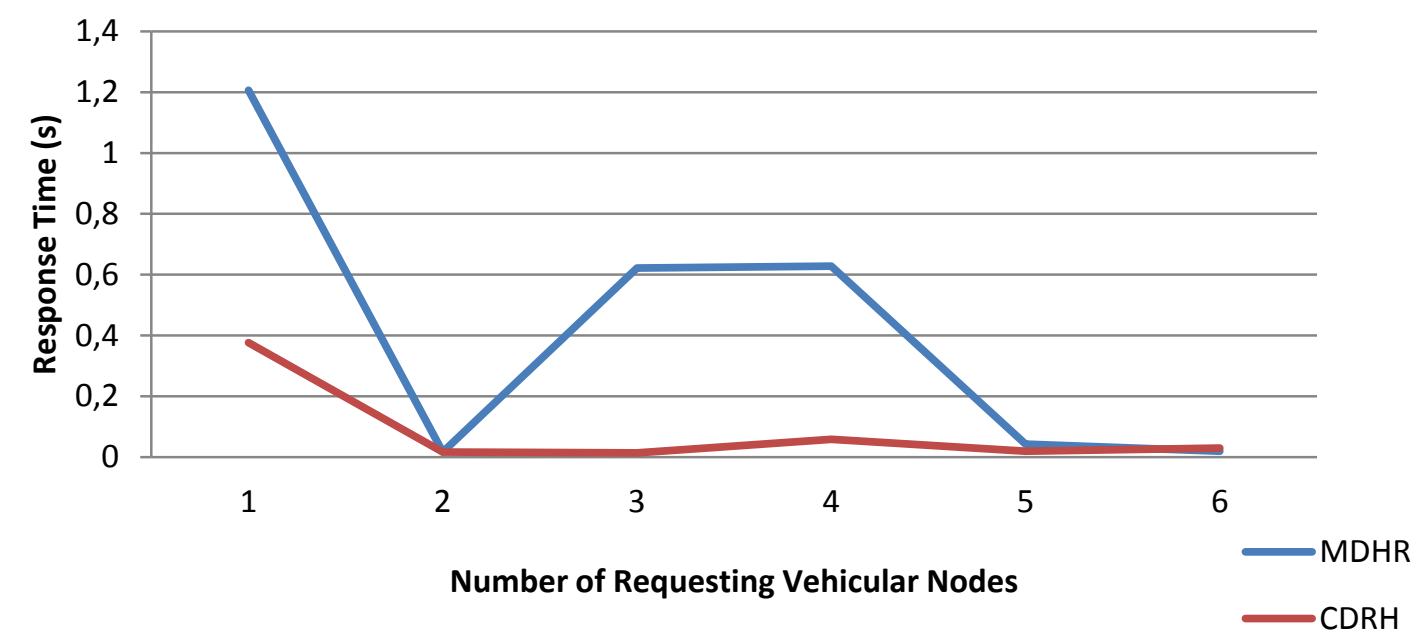

Figure 10. Response Time / Number of Vehicular Nodes Requesting for Data.

Figure 10 demonstrates the advantage of our solution over MDHR in term of time of response. Because of the packet (request and data) dissemination mode used in our solution, the data replication, and the opportunistic communication between vehicular nodes, CDRH outperforms MDHR.

The data collection technique used in MDHR can affect the collect time, packets overhead and also the efficiency (rate of successful requests) if the distance separating between the node holding the data and the RS node is important.

One very important prerequisite for the successful and also sustainable deployment of VSNs is the scalability of the applied information dissemination scheme. The autonomously acting network must ensure delivery of data to those nodes which are interested in it with low latency, while efficiently leveraging network resources.

For the scalability measure, we vary the number of nodes and the surface of the network keeping one density $\left(475^{*} 10^{-4}\right)$. 
Table 2. Number of Nodes per Surface.

\begin{tabular}{|c|c|c|c|}
\hline $\begin{array}{c}\text { Number of } \\
\text { sensor nodes }\end{array}$ & $\begin{array}{c}\text { Number of } \\
\text { vehicular nodes }\end{array}$ & $\begin{array}{c}\text { Total number of } \\
\text { nodes }\end{array}$ & Surface \\
\hline 55 & 40 & 95 & $1000 * 2000$ \\
\hline 110 & 80 & 190 & $2000 * 2000$ \\
\hline 121 & 88 & 209 & $2200 * 2000$ \\
\hline 132 & 96 & 228 & $2400 * 2000$ \\
\hline 154 & 112 & 266 & $2800 * 2000$ \\
\hline 165 & 120 & 285 & $3000 * 2000$ \\
\hline
\end{tabular}

In figures 12, 13 and 14, we evaluate the scalability of CDRH. The x-axis was configured to the number of nodes in the network, which varies from 95 to 285 . The number of requesting vehicles is set to 2 .

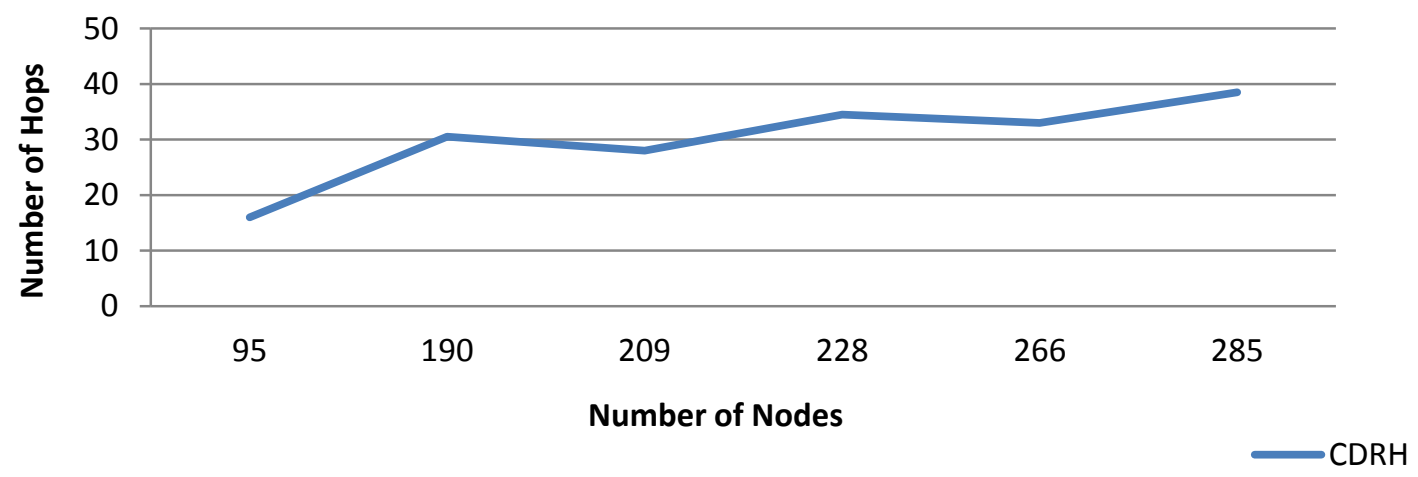

Figure 12. Number of Hops / Number of Nodes.

An increase of number of hops in the proposed scheme as the number of nodes increase is observed in figure 12. That's due to the fact that more the target surface is large, more the distance separating the requesting vehicular node and a node containing the data (node source or a node containing a replicas of the requested data) is important.

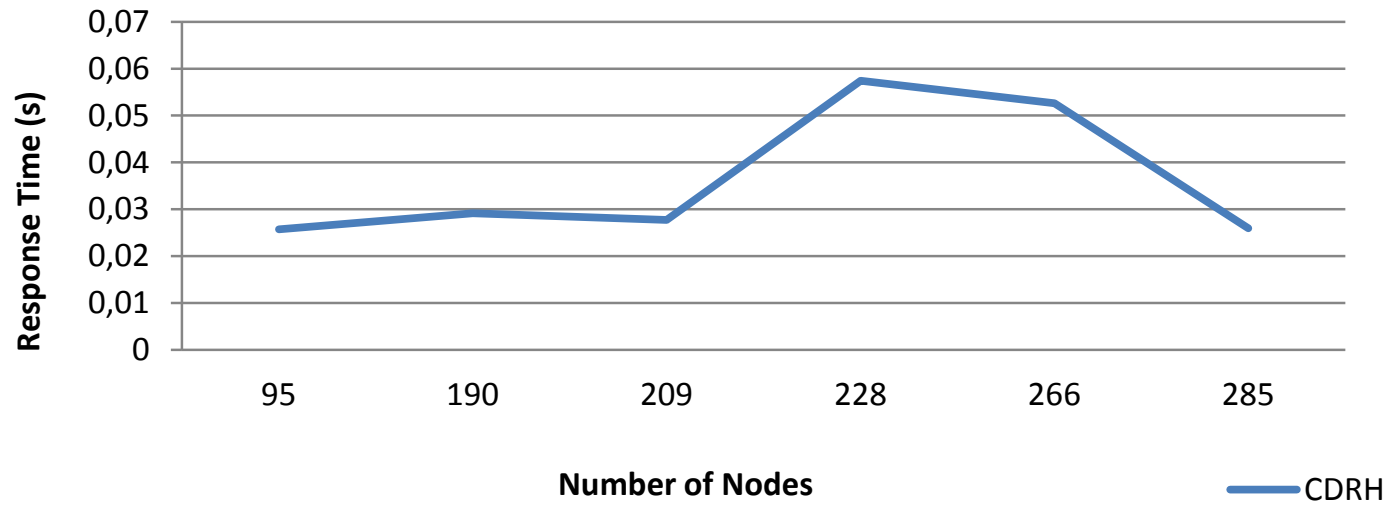

Figure 13. Time of Response / Number of Nodes. 
By increasing the number of nodes as the surface increase, the response time stays stable between 0,025 and 0,057 . CDRH is suitable for large networks as we can saw in figure 13 that the response time decreases when the number of nodes is set to 266 and 285 .

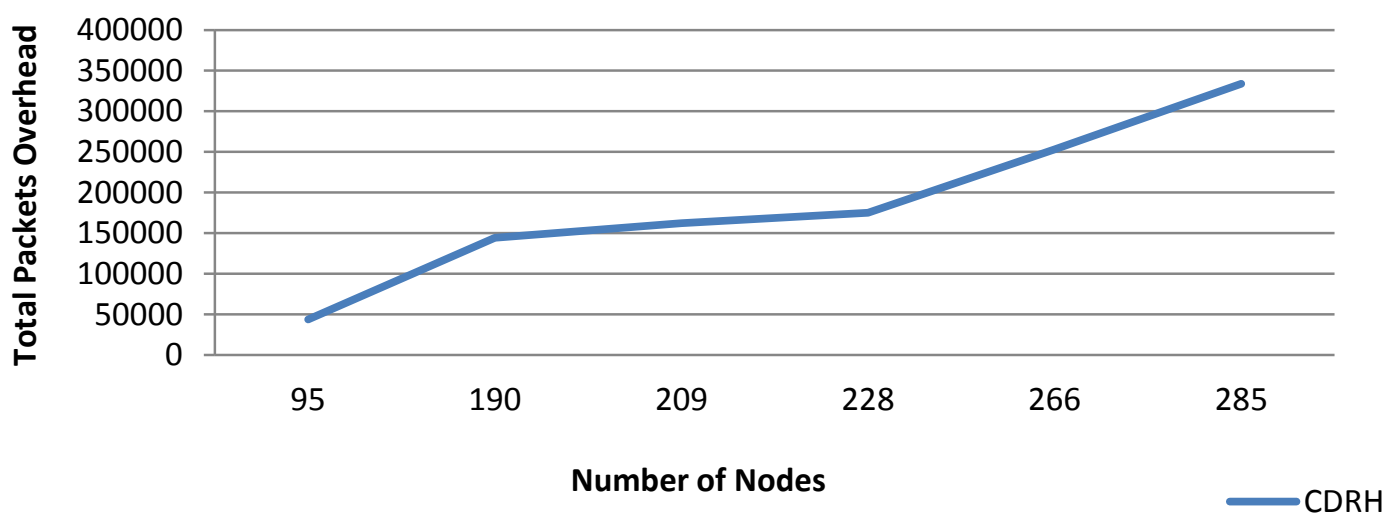

Figure 14. Total Packets Overhead / Number of Nodes.

In figure 14, more the target surface is large, more the traffic is important, that is due mainly to the fact that the number of beacon messages increase as the number of nodes increase.

Scalability results show that CDRH protocol offers good performances. CDRH has the ability to handle the addition of nodes without suffering a noticeable loss in performance or increase in administrative complexity.

\section{Conclusion}

In this paper, a new data dissemination and harvesting system CDRH based on geographical dissemination and data replication mechanism on vehicular sensor networks is proposed. Designed for hybrid VSN architecture, it is a collaborative protocol which allows communication between vehicles and between vehicles and road infrastructure. CDRH use a hybrid data collection, where a request-driven data collection is used by vehicular nodes and a specific algorithm which is a permanent data collection to optimize the packets retransmission within intersections that makes it suitable for both highways and urban environments.

The aim of CDRH is to deliver a requested data and make this data accessible for other requesting vehicles. Simulation results of CDRH demonstrate the advantage of using the data replication process, the communication between vehicles and the dissemination strategy used to overcome collisions and overhead in the network. We are currently extending this work by adding new disseminations mode "clusters-based dissemination" and "opportunistic dissemination", data aggregation, meta-data dissemination, and an extensive simulation in order to do a compromise between delivery time and overhead in the network. However, more comprehensive simulation study needs to be conducted to prove the advantage of the protocol. 


\section{References}

[1] A. P.Sistla, O. Wolfson, and B. Xu. "Opportunistic Data Dissemination in Mobile Peer-toPeer Networks". International Symposium on Spatial and Temporal Databases, Angra dos Reis, Brazil, 2005.

[2] R. Adler, P. Buonadonna, J. Chhabra, M. Flanigan, L. Krishnamurthy, N. Kushalnagar, L. Nachman, and M. Yarvis. "Design and Deployment of Industrial Sensor Networks: Experiences from the North Sea and a Semiconductor Plant”. ACM SenSys, 2005.

[3] A. Mainwaring, J. Polastre, R. Szewczyk, and D. Culler. "Wireless Sensor Networks for Habitat Monitoring". WSNA, 2002.

[4] A. Cerpa, J. Elson, D. Estrin, L. Girod, M. Hamilton, and J. Zhao. "Habitat monitoring: Application driver for wireless communications technology". ACM SIGCOMM Workshop on Data Comm in Latin America and the Caribbean, 2001.

[5] U. Lee, E. Magistretti, B. Zhou, M. Gerla, P. Bellavista, and A. Corradi. "Efficient Data Harvesting in Mobile Sensor Platforms". IEEE PerSeNS'06 Workshop, March 2006.

[6] I. Salhi, M.O. Cherif, and S.M. Senouci. "A New Architecture for Data Collection in Vehicular Networks". IEEE, 2009.

[7] U. Lee, and M. Gerla. "A survey of urban vehicular sensing platforms". Computer networks, Advances in Wireless and Mobile Networks 54 (2010) 527-544.

[8] B. Hull, V. Bychkovsky, Y. Zhang, K. Chen, M. Goraczko, A. Miu, E. Shih, H. Balakrishnan and S. Madden. "CarTel: A Distributed Mobile Sensor Computing System", 2006.

[9] S.Y. Ni, Y.C. Tseng, Y.S. Chen and J. sheu. 'The Broadcast storm problem in a mobile ad hoc networks' $.5^{\text {th }}$ Annual ACM/IEEE International Conference on mobile computing and Networking, Seattle, Washington, August 1999.

[10] L. Kheroua, S. Moussaoui, and L. Mansour. "An Agent based Rumor Dissemination for Routing in Wireless Sensor Networks”, ISPS, Algeria, 2011.

[11] D. Iabbassen, S. Moussaoui. "An Energy Saving Data Dissemination Protocol for Wireless Sensor Networks”. R. Benlamri (Ed.): NDT 2012, Part I, CCIS 293, pp. 470-482, Springer-Verlag Berlin Heidelberg, 2012.

[12] L. Yang, J. Xu, G. Wu, and J. Guo. "Road Probing: RSU Assisted Data Collection in Vehicular Networks". IEEE, 2009.

[13] K.W. Lim, W.S. Jung, and Y.B. Ko. "Multi-Hop Data Dissemination with Replicas in Vehicular Sensor Networks", Proceeding Vehicular Technology Conference, 2008. VTC Spring 2008. IEEE, 11-14 May 2008, page(s): 3062 - 3066, Singapore, ISSN: 1550-2252, ISBN: 978-1-4244-1644-8.

[14] S. Dornbush, and A. Joshi. "Street Smart Traffic: Discovering and Disseminating Automobile Congestion using VANETs", Proc. of the IEEE VTC, Spring, April (2007).

[15] A. Vegni, M. Biagi, and R. Cusani. "Smart Vehicles, Technologies and Main Applications in Vehicular Ad hoc Networks". http://dx.doi.org/10.5772/55492.

[16] A. Seth, P. Darragh, S. Liang, Y. Lin, and S. Keshav. "An Architecture for Tetherless Communication". DTN Workshop, 2005.

[17] H. Alshaer, and E. Horlait. "An Optimized Adaptive Broadcast Scheme for Inter-vehicle Communication". Proceedings of the IEEE Vehicular Technology Conference (IEEE 
VTC2005-Spring), pages 2840- 2844, Stockholm, Sweden, May 2005. http://dx.doi.org/10.1109/VETECS.2005.1543865.

[18] A. Benslimane. "Optimized Dissemination of Alarm Messages in Vehicular Ad-hoc

Networks (VANET)". In Proceedings of the 7th IEEE International Conference,Toulouse, France, June 2004. http://dx.doi.org/10.1007/978-3-540-25969-5_59.

[19] J. Nzoonta, and C. Borcea. "STEID: A protocol for emergency information dissemination in vehicular networks", 2006.

[20] A. Agarwal, and T. Little. "Opportunistic Networking in Delay Tolerant Vehicular Ad Hoc Networks". Advances in Vehicular Ad-Hoc Networks: Developments and Challenges (Ed. M. Watfa), 2010.

[21] H. Wu, R. Fujimoto, R. Guensler and M. Hunter. "MDDV: A Mobility centric Data Dissemination Algorithm for Vehicular Networks". Proceedings of the Proceedings of the 1st ACM International Workshop on Vehicular Ad Hoc Networks (VANET '04), pages 47-56, NewYork, NY, USA, 2004.

[22] L. Bononi, M. Di Felice. "A Cross Layered MAC and Clustering Scheme for Efficient Broadcast in VANETs". IEEE MASS'07, October 2007, Pisa, Italy. http://dx.doi.org/10.1109/MOBHOC.2007.4428735.

[23] F. Wang, D. Zeng, and L. Yang, "Smart cars on smart roads: an IEEE intelligent transportation systems society update". IEEE Pervasive Computing, Vol. 5, No. 4, 2006.

[24] N. Haddadou, A. Rachedi, and Y. Ghamri-Doudane. "Modeling and Performance Evaluation of Advanced Diffusion with Classified Data in Vehicular Sensor Networks". Wireless Communications and Mobile Computing 11, 12 (2011) 1689-1701", 2011. http://dx.doi.org/10.1002/wcm.1220.

[25] C.E. Palazzi, F. Pezzonib, and P.M. Ruizc. "Delay-bounded data gathering in urban vehicular sensor networks". Pervasive and Mobile Computing , 2011. http://dx.doi.org/10.1016/j.pmcj.2011.06.008.

[26] A. Skordylis, N. Trigoni. "Delay-bounded routing in vehicular ad-hoc networks". Proceedings of the 9th ACM international symposium on Mobile ad hoc networking and computing, MobiHoc'08, ACM, New York, NY, USA, 2008, pp. 341-350. http://dx.doi.org/10.1145/1374618.1374664.

[27] M.T. Sun, W. Feng, T. Lai, K. Yamada, H. Okada, and K. Fujimura. "GPS-based message broadcast for adaptive inter-vehicle communications". Proceedings of the IEEE Vehicular Technology Conference (VTC 2000), vol.1: 2685-2692, Boston, USA, September 2000 .

[28] F. Wang, D. Zeng, and L. Yang. "Smart cars on smart roads: an IEEE intelligent transportation systems society update". IEEE Pervasive Computing, Vol. 5, No. 4, 2006.

[29] ns-2 (The Network Simulator), http://www.isi.edu/nsnam/ns.

[30] D. Krajzewicz, and C. Rossel. "Simulation of Urban Mobility (SUMO)". German Aerospace Centre, 2007. Available at http://sumo.sourceforge.net/index.shtml.

[31] MOVE (MObility model generator for VEhicular networks): Rapid Generation of Realistic Simulation for VANET, 2007. Available at http://lens1.csie.ncku.edu.tw/MOVE/index.htm. 


\section{Copyright Disclaimer}

Copyright reserved by the author(s).

This article is an open-access article distributed under the terms and conditions of the Creative Commons Attribution license (http://creativecommons.org/licenses/by/3.0/). 